\title{
Maximal sum-free sets in finite abelian groups, II
}

\author{
H.P. Yap
}

\begin{abstract}
Maximal sum-free sets in groups $z_{n}$, where $n$ is any positive integer such that every prime divisor of $n$ is congruent to 1 modulo 3 , are completely characterized.
\end{abstract}

Let $G$ be an additive group. If $S$ and $T$ are non-empty subsets of $G$, we write $S \pm T$ for $\{s \pm t ; s \in S, t \in T\}$ respectively, $|S|$ for the cardinality of $S$ and $\bar{S}$ for the complement of $S$ in $G$. We say that $S$ is sum-free in $G$ if $S$ and $S+S$ have no common element and that $S$ is maximal sum-free in $G$ if $S$ is sum-free in $G$ and $|S| \geq|T|$ for every $T$ sum-free in $G$. We denote by $\lambda(G)$ the cardinality of a maximal sum-free set in $G$. We say that $S$ is in a.p. (arithmetic progression) with difference $d$ if $s=\{s, s+d, \ldots, s+n d\}$ for some $s, d \in G$ and some integer $n \geq 0$. We say that $s$ is quasi-periodic if there exists a subgroup $H$, of order $\geq 2$, of $G$ such that $S$ is the disjoint union of a non-empty set $S^{\prime}$ consisting of $H$-cosets and a residue set $S^{\prime \prime}$ contained in a remaining $H$-coset. We say that a prime $p$ is a bad prime if $p$ is congruent to 1 modulo 3 .

Erdös [2] gives certain upper and lower bounds for $\lambda(G)$ of finite abelian groups $G$. Exact values $\lambda(G)$ for all finite abelian groups $G$, except when every prime divisor of $|G|$ is bad, were determined by Diananda and Yap [1]. In this exceptional case,

$$
|G|(m-1) / 3 m \leq \lambda(G) \leq(|G|-1) / 3
$$

where $m$ is the exponent of $G$. For elementary abelian p-groups $G$ of 
order $p^{n}$, Rhemtulla and Street [5] prove that $\lambda(G)=k p^{n-1}$, where $p=3 k+1$ is a prime.

The structure of maximal sum-free sets in the following groups were completely characterized:

(i) $G$ is any abelian group such that $|G|$ has a prime divisor congruent to 2 modulo $3[1,7]$;

(ii) $G=Z_{p}$ where $p$ is a bad prime $[8,5]$;

(iii) $G$ (abelian and non-abelian) is of order $3 p$, where $p$ is a bad prime [9];

(iv) $G$ is an elementary abelian p-group where $p$ is a bad prime [6];

(v) $G$ is an elementary abelian 3-group and $G=z_{3} \oplus z_{3} \oplus z_{p}$ where $p$ is a bad prime [10].

We shall apply a Lemma in [5] and Theorem 2.1 in [3], which are restated respectively as Lemma 1 and Theorem 1 here, to prove Theorem 2 which generalizes some results in [8] and [5].

LEMMA 1. Let $G=z_{n}, n=3 k+1$ and $S$ be a sum-free set in $G$ satisfying $|S|=k,-S=S$ and $\bar{S}=S+S$. Then

(i) if $|(S+g) n S|=1$ for some $g$ in $G$, then $\left|\left(S+g^{*}\right) n S\right| \geq k-3$ where $g^{*}=3 g / 2$ and $\pm g / 2 \in S$;

(ii) if $|(S+g) \cap S|=\lambda>1$ for some $g \neq 0$ in $G$, then $g^{*}=s_{1}-s_{2}$, where $s_{1}, s_{2}\left(\neq s_{1}\right) \in S$ and $s_{1}+g, s_{2}+g \in s$, is such that $\left|\left(S+g^{*}\right) \cap S\right| \geq k-(\lambda+1)$.

THEOREM 1 (Kemperman). Let $G$ be an abelian group with subsets $A$ and $B$ such that $|A|,|B| \geq 2$. If $|A+B|=|A|+|B|-1$, then either $A+B$ is in a.p. or $A+B$ is quasi-periodic.

We first prove the following lemmas.

LEMMA 2. Let $G=z_{n}$ where $n$ is any positive integer such that every prime divisor $p$ of $n$ is bad. If $S$ is a maximal sum-free set in $G$, then 
(i) if $-S \neq S,\left|S+S^{*}\right|=|S|+\left|S^{*}\right|-1$, where $S^{*}=-S \cup S$;

(ii) if $-S=S$, either $|S+S|=2|S|-1$ or $\bar{S}=S+S$.

Proof. By Kneser's Theorem [4], there exists a subgroup $K$ of $G$ such that $S+S+K=S+S$ and $|S+S| \geq 2|S+K|-|K|$. It is clear that $K$ is a proper subgroup of $G$.

Suppose that $|k|=q>1$. Let $n=3 k+1=p q, p=3 r+1$, $q=3 s+1$. Then $\lambda(G)=k=r q+s$ and

$$
|G|-|S|=2 k+1 \geq|S+S| \geq 2(k / q] q-q
$$

where ( $x$ ] denotes the smallest positive integer $\geq x$.

Thus $2 k+1 \geq|S+S| \geq(2 x+1) q$, which is impossible. Hence $|S+S| \geq 2|S|-1$.

If $-S=S$, then $|S+S|$ is odd and from $2 k+1 \geq|S+S| \geq 2 k-1$, it follows that either $|S+S|=2|S|-1$ or $|S+S|=2|S|+1$ and thus $\bar{S}=S+S$.

If $-S \neq S$, then again by Kneser's Theorem there exists a proper subgroup $K$ of $G$ such that $S+S^{*}+K=S+S^{*}$ and $\left|S+S^{*}\right| \geq|S+K|+\left|S^{*}+K\right|-|K|$.

In this case, we can show that $|K|=1$. Thus

$2 k+1 \geq\left|S+S^{*}\right| \geq|S|+\left|S^{*}\right|-1 \geq|S|+(|S|+2)-1=2 k+1$. Hence $\left|S^{*}\right|=|S|+2$ and $\left|S+S^{*}\right|=|S|+\left|S^{*}\right|-1$.

The proof of Lemma 2 is now complete.

LEMMA 3. Let $G=z_{n}$ where $n$ is any positive integer such that every prime divisor $p$ of $n$ is bad. Let $S$ be a maximal sum-free set in $G$.

(I) If $-S \neq S$, then $S$ can be mapped onto $\{k, k+1, \ldots, 2 k-1\}$ under an automorphism of $G$.

(II) If $-S=S$ and $|S+S|=2|S|-1$, then $S$ can be mapped onto $\{k+1, k+2, \ldots, 2 k\}$ under an automorphism of $G$.

Proof (I). If $-S \neq S$, then by Lemma 2, $\left|S+S^{*}\right|=|S|+\left|S^{*}\right|-1$. By Kemperman's Theorem, we have either $S+S^{*}$ is in a.p. or $S+S^{*}$ is quasi-periodic. 
Suppose that $S+S^{*}$ is quasi-periodic, then from $\bar{S}=S+S^{*}$ it follows that $S$ is also quasi-periodic. Thus $S^{\circ}$, which is a subset of $S$ consisting of $H$-cosets, will be a maximal sum-free set in $G / H$ while the non-empty residue set $S^{\prime \prime}$ which is contained in a remaining $H$-coset will violate the sum-free property of $S$. Hence $S+S^{*}$ cannot be quasi-periodic.

Let $S+S^{*}=\left\{a^{\prime}+i d ; i=0,1, \ldots, 2 k\right\}$. Since $\left|S+S^{*}\right|=2 k+1$, therefore $(d, n)=1$ (the g.c.d. of $d$ and $n$ ). Hence under an automorphism of $G$, we can write $S+S^{*}=\{a+i ; i=0,1, \ldots, 2 k\}$. Then $S=\overline{S+S^{*}}=\{a+i ; i=2 k+1, \ldots, 3 k\}$. From $\left|S^{*}\right|=|S|+2$, we have either

(i) $2 a+2 k+3+3 k \equiv 0(\bmod n)$, that is $a \equiv-(k+1)(\bmod n)$, or

(ii) $2 a+2 k+1+3 k-2 \equiv 0(\bmod n)$, that is $a \equiv-(k-1)$ $(\bmod n)$.

(i) gives the maximal sum-free set $S=\{k, k+1, \ldots, 2 k-1\}$.

(ii) gives $S=\{k+2, k+3, \ldots, 2 k+1\}$ which can be mapped onto $\{k, k+1, \ldots, 2 k-1\}$ under an automorphism of $G$.

Proof (II). Applying similar methods we can show that under an automorphism of $G, S+S$ can be mapped onto $S+S=\{a+i ; i=0,1, \ldots, 2 k-2\}$. Since $-S=S$, therefore $2 a+2 k-2 \equiv 0(\bmod n)$, that is $a \equiv-(k-1)$, (mod $n)$.

Then $s+s=\{-(k-1),-(k-2), \ldots, k-1\}$, and

$$
S \subseteq \overline{S+S}=\{k, k+1, \ldots, 2 k+1\} \text {. }
$$

But $2 k=k+. k \$ S+S$, therefore $k \notin S$. Hence $S=\{k+1, \ldots, 2 k\}$.

The proof of Lemma 3 is now complete.

LEMMA 4. Let $G=z_{n}, n=3 k+1$ and $S$ be a sum-free set in $G$ satisfying $|S|=k,-S=S$ and $\bar{S}=S+S$. Then $|(S+g) \cap S|>1$ for every $g \in \bar{S}$ with $(g, n)>1$.

Proof. We first note that $(S+g) \cap S \neq \varnothing$ if and only if $g \notin S$. Suppose that $|(S+g) n S|=1$ for some $g \in \bar{S}$ with $(g, n)>1$. Then 
by Lemma $1,|(S+f) n s| \geq k-3$ where $f=3 g / 2$.

Now $|(S+f) n S| \neq k$, since $S$ cannot be a union of cosets of a nontrivial subgroup of $G$. Thus $|(S+f) \cap S|=k-1, k-2$ or $k-3$.

Let $H=[f]$, the subgroup of $G$ generated by $f$, where $|H|=p=3 r+1>1, p q=n, q=3 s+1,|S|=s p+r$.

(i) If $|(S+f) \cap S|=k-1$, then

$$
S=U H_{i} \cup\left\{a_{1}, a_{1}+f, \ldots, a_{1}+m_{1} f\right\}
$$

where each $H_{i}$ is a coset of $H,\left|\cup H_{i}\right|=s p$ and $m_{1}=r-1$. In this case it is clear that $S^{\prime \prime}=\left\{a_{1}, a_{1}+f, \ldots, a_{1}+m_{1} f\right\} \subseteq H$. But $H \subseteq S+S$ which contradicts the fact that $(S+S) \cap S=\emptyset$.

$$
\begin{aligned}
& \text { (ii) If }|(S+f) n S|=k-2 \text {, then } \\
& S=U H_{i} \cup\left\{a_{1}, a_{1}+f, \ldots, a_{1}+m_{1} f\right\} \cup\left\{a_{2}, a_{2}+f, \ldots, a_{2}+m_{2} f\right\}, m_{1} \leq m_{2} .
\end{aligned}
$$$$
\text { Since }-S=S, s \geq 2 \text {, therefore } H \subseteq S+S \text {, and }
$$$$
-\left\{a_{1}, a_{1}+f, \ldots, a_{1}+m_{1} f\right\}=\left\{a_{2}, a_{2}+f, \ldots, a_{2}+m_{2} f\right\} .
$$

Hence $m_{1}+m_{2}$ is even. If $\left|\mathrm{UH}_{i}\right|=(s-1) p$, then $m_{1}+m_{2}=p+r-2$ is odd, which is impossible. Hence $\left|U H_{i}\right|=s p$ and $m_{1}+m_{2}=r-2$.

But then

$$
\left\{a_{1}, \ldots, a_{1}+m_{1} f, a_{2}, \ldots, a_{2}+m_{2} f\right\}+\left\{a_{1}, \ldots, a_{1}+m_{1} f, a_{2}, \ldots, a_{2}+m_{2} f\right\}
$$

contains elements from 3 distinct cosets of $H$, which contradicts the fact that $\bar{S}=S+S$.

$$
\begin{aligned}
& \text { (iii) If }|(S+f) \cap s|=k-3 \text {, then } \\
& s=U H_{i} \cup\left\{a_{1}, \ldots, a_{1}+m_{1} f, a_{2}, \ldots, a_{2}+m_{2} f, a_{3}, \ldots, a_{3}+m_{3} f\right\}, \\
& \qquad m_{1} \leq m_{2} \leq m_{3} .
\end{aligned}
$$

Suppose that $S \cap H=\emptyset$. Then from $-S=S$ we know that

$$
\left\{a_{1}, \ldots, a_{1}+m_{1} f, a_{2}, \ldots, a_{2}+m_{2} f, a_{3}, \ldots, a_{3}+m_{3} f\right\}
$$

is contained in exactly two distinct cosets of $H$. Without loss of generality, assume that $a_{2} \in a_{1}+H$. Then 


$$
-\left\{a_{1}, \ldots, a_{1}+m_{1} f, a_{2}, \ldots, a_{2}+m_{2} f\right\}=\left\{a_{3}, \ldots, a_{3}+m_{3} f\right\}
$$

which is impossible, because the right hand side is in a.p. with difference $f$ while the left hand side is not in a.p. with difference $f$. Hence $S \cap H \neq \emptyset$. But then $\left|\cup H_{i}\right|=0$ and $s=2$, $m_{1}+m_{2}+m_{3}=2 p+r-3$. In this case, $s+s$ will contain 5 distinct full cosets of $H$ which is impossible.

The proof of Lemma 4 is now complete.

THEOREM 2. Let $G=Z_{n}$ where $n$ is any positive integer such that every prime divisor of $n=3 k+1$ is bad. If $S$ is a maximal sum-free set in $G$, then $S$ can be mopped, under an automorphism of $G$, to one of the following:

(i) $\{k+\cdot 1, k+2, \ldots, 2 k\}$;

(ii) $\{k, k+1, \ldots, 2 k-1\}$;

(iii) $\{k, k+2, k+3, \ldots, 2 k-1,2 k+1\}$.

Proof. By Lemmas 2 and 3 , it remains to show that if $-S=S$, $\bar{S}=s+S$, then $s$ can be mapped to $\{k, k+2, k+3, \ldots, 2 k-1,2 k+1\}$ under an automorphism of $G$. The method used here is a modification of a method due to Rhemtulla and Street [5].

If $|(S+g) \cap S|=2$ for some $g \in G$ such that $(g, n)=1$, then by the same method as the proof of Theorem 2 in [5], we can show that under an automorphism of $G, S$ can be mapped onto $\{k, k+2, k+3, \ldots, 2 k-1,2 k+1\}$.

We are now left with the case where $S$ satisfies the conditions of Lemma 1 and $|(S+g) n S| \neq 1$ for any $g$ in $G$ satisfying $(g, n)=1$. If $|(S+g) n S|$ is maximal for some $g$ satisfying $(g, n)=1$, then by taking an automorphism of $G$ if necessary, assume that $|(S+1) n S|$ is maximal. We write

$$
s=\left\{a_{1}, \ldots, a_{1}+m_{1}, a_{2}, \ldots, a_{2}+m_{2}, \ldots, a_{h}, \ldots, a_{h}+m_{h}\right\},
$$

where $1<a_{1} \leq a_{1}+m_{1}<a_{2}-1<a_{2}+m_{2}<\ldots<a_{h}-1<a_{h}+m_{h}<n$, and $a_{i}, \ldots, a_{i}+m_{i}$ denotes a string of $\left(m_{i}+1\right)$ consecutive elements of $S$. 
We have

(2) $|(S+1) n S|=k-h \geq|(S+g) n S|$ for every $g \neq 0$ in $G$.

Hence $h$ is minimal in (I).

Let $X=\left\{a_{1}, a_{2}, \ldots, a_{h}\right\}$. Then

$$
Y=\left\{a_{1}+m_{1}+1, \ldots, a_{h}+m_{h}+1\right\}=1-X,
$$

since $-S=S$.

For each $i=1, \ldots, h, a_{i}-1 \notin S$. Since $\bar{S}=S+S$ and $|(S+g) \cap S| \geq 2$ for any $g(\neq 0) \in \bar{S}$ (by assumption and Lemma 4$)$, therefore there exist $s_{1}, s_{2}\left(\neq s_{1}\right)$ in $s$ such that $a_{i}-1=s_{2}-s_{1}$ and $g=-s_{1}-s_{2} \neq 0$. We have now $s_{1}+g, s_{2}+g \in S$ and $k-h \geq|(S+g) \cap S| \geq 2$, therefore, by Lemma 1, we have $\left|\left(S+a_{i}-1\right) n s\right| \geq h-1$. But for any $s_{1}, s_{2} \in S, s_{1}+a_{i}-1=s_{2}$ implies that $s_{1} \in X, s_{2} \in-X$ and $s_{1}+a_{i} \in Y$. Hence

$$
h \geq\left|\left(X+a_{i}\right) \cap Y\right| \geq h-1 \text { for all } i=1, \ldots, h \text {. }
$$

Suppose that $h \geq 3$.

If for each $j=1, \ldots, h, X+a_{j}=Y=1-X$, then $X+[X-X]=X, h=|X|=|[X-X]|=p$, which divides $n$, and

$$
2 \sum_{i=1}^{h} a_{i}+h a_{j} \equiv h(\bmod n) \text { for each } j=1, \ldots, h \text {. }
$$

Thus

$$
h\left(a_{i}-a_{j}\right) \equiv 0(\bmod n) \text { for every } i, j=1, \ldots, h .
$$

If $n$ is a prime, we already get a contradiction here. Otherwise, $X=a+H$ where $H=[q], p q=n$. We then have

$$
a_{1}=a, a_{2}=a+q, \ldots, a_{p}=a+(p-1) q .
$$

Substituting (6) into (4) for $j=1$, we get $(3 a-1) p \equiv 0$ (mod $n)$ from which it follows that $a=2 s+1(q=3 s+1)$ and

$$
S=\left\{2 s+1, \ldots, 2 s+1+m_{1}, \ldots, 2 s+1+(p-1) q+m_{p}\right\} .
$$


But $2 s+1+2 s+1+(p-1) q+m_{p}>n$ which contradicts the fact that $a_{1}+a_{p}+m_{p}=n$. Hence, for at least one $t \in\{1, \ldots, h\}$, $\left|\left(X+a_{t}\right) \cap Y\right|=h-1$.

If there is only one $t \in\{1, \ldots, h\}$ such that $\left|\left(X+a_{t}\right) \cap Y\right|=h-1$, then there are at least two distinct $i, j \in\{1, \ldots, h\}$ such that $X+a_{i}=Y=X+a_{j}$ and thus $X+a_{i}-a_{j}=X$ from which it follows that $X$ is the union of cosets of a nontrivial subgroup of $G$. (If $n$ is a prime, we get a contradiction here.) Thus $\left|\left(X+a_{t}\right) \cap Y\right| \neq h-1$ which contradicts the hypothesis.

Hence there are at least two $t_{1}, t_{2} \in\{1, \ldots, h\}$ such that $\left|\left(X+a_{t_{1}}\right) \cap Y\right|=h-1=\left|\left(X+a_{t_{2}}\right) \cap Y\right|$. Then

(7) $\left\{a_{1}, \ldots, a_{t-1}, a_{t+1}, \ldots, a_{h}\right\}+a_{t}=$

$$
1-\left\{a_{1}, \ldots, a_{t-1}, a_{t+1}, \ldots, a_{h}\right\}, t=t_{1}, t_{2},
$$

from which it follows that

$$
2 \sum_{i=1}^{h} a_{i}+(h-3) a_{t} \equiv h-1(\bmod n), t=t_{1}, t_{2},
$$

and thus

$$
(h-3)\left(a_{t_{1}}-a_{t_{2}}\right) \equiv 0 \quad(\bmod n) \text {. }
$$

Suppose there are also at least two $r_{1}, r_{2} \in\{1, \ldots, h\}$ such that $\left|\left(X+a_{r_{i}}\right) \cap Y\right|=h$. Divide $\{1, \ldots, h\}$ into the union of two disjoint subsets $R=\left\{r_{1}, \ldots, r_{u}\right\}, u \geq 2, T=\left\{t_{1}, \ldots, t_{v}\right\}, v \geq 2$ such that $\left|\left(X+a_{r_{i}}\right) \cap Y\right|=h$ and $\left|\left(X+a_{t_{i}}\right) \cap Y\right|=h-1$. Then

$$
\begin{gathered}
h\left(a_{r}-a_{r^{\prime}}\right) \equiv 0(\bmod n) \text { for every } r, r^{\prime} \in R, \\
(h-3)\left(a_{t}-a_{t^{\prime}}\right) \equiv 0(\bmod n) \text { for every } t, t^{\prime} \in T .
\end{gathered}
$$

Let 


$$
\begin{aligned}
& a_{t_{1}}+a_{r_{1}} \equiv 1-a_{p_{1}}(\bmod n), \\
& a_{t_{2}}+a_{r_{1}} \equiv 1-a_{p_{2}}(\bmod n) .
\end{aligned}
$$

Then $a_{t_{1}}-a_{t_{2}} \equiv a_{p_{2}}-a_{p_{1}}(\bmod n)$ from which it follows that at least one of $p_{1}, p_{2}$ is in $T$. Suppose that $p_{1}=t \in T$. Let

$$
a_{t_{1}}+a_{r_{2}} \equiv 1-a_{p_{3}}(\bmod n) \text {. }
$$

Then from (12) and (14), we have $a_{r_{1}}-a_{r_{2}} \equiv a_{p_{3}}-a_{t}(\bmod n)$ and thus $p_{3}=r \in R$. Then $h\left(a_{r}-a_{t}\right) \equiv 0 \quad(\bmod n)$, and thus from (10), we have

$$
h\left(a_{r}-a_{t}\right) \equiv 0 \quad(\bmod n) \text { for every } r \in R \text {. }
$$

Let

$$
a_{t_{2}}+a_{r_{2}} \equiv 1-a_{p_{4}}(\bmod n) \text {. }
$$

Then from (14) and (16), we have $a_{t_{1}}-a_{t_{2}} \equiv a_{p_{4}}-a_{r}(\bmod n)$ from which it follows that $p_{4}=t^{\prime} \in T$. Hence $(h-3)\left(a_{t^{\prime}}-a_{r}\right) \equiv 0(\bmod n)$. Then from (11), we have

$$
(h-3)\left(a_{t}-a_{p}\right) \equiv 0 \quad(\bmod n) \text { for every } t \in T \text {. }
$$

But (15) and (17) cannot occur at the same time. Hence for at most one $j \in\{1, \ldots, h\},\left|\left(X+a_{j}\right) \cap Y\right|=h$. But then (9) is true for every $t_{1}, t_{2} \in\{1,2, \ldots, j-1, j+1, \ldots, h\}$. We have either

(i) $h-3=v p>0, p \mid n,(v, n)=1$ and

$$
X^{\prime}=\left\{a_{1}, \ldots, a_{j-1}, a_{j+1}, \ldots, a_{h}\right\}=a+A^{\prime}
$$

where $A^{\prime} \subseteq H=[q], p q=n$, which is impossible because $h-1=v p+2>|G / H| ;$ or

(ii) $h=3$ and thus

$$
\begin{aligned}
& S=\{a, \ldots, a+c-1, k+c+1, \ldots, 2 k-c, 3 k+2-a-c, \ldots, 3 k+1-a\} \\
& \text { where } a \leq k \text { and } c<k / 2 .
\end{aligned}
$$

Then from (8) we get

$$
0 \equiv 3-1-2\{a+k+c+1-(a+c-1)\} \quad(\bmod n),
$$


that is $1 \equiv k+2(\bmod n)$ which is impossible.

Thus $h \leq 2$. But $h \neq 1$, because $S$ is not in a.p. If $h=2$, then $S=\{ \pm k / 2, \pm(1+k / 2), \ldots, \pm(k-1)\}$ which maps, under an automorphism of $G$, to $\{k, k+2, k+3, \ldots, 2 k-1,2 k+1\}$.

Finally, suppose that $|(S+g) n S| \geq 2$ for every $g \neq 0$ in. $G$ with $(g, n)=1$ and that $|(s+g) n S|$ is maximal for some $g$ in $G$ with $(g, n)>1$. By taking an automorphism of $G$, if necessary, suppose that $g \mid n$. Then we can write

$$
S=U H_{i} \cup\left\{a_{1}, a_{1}+g, \ldots, a_{1}+m_{1} g, \ldots, a_{h}, a_{h}+g, \ldots, a_{h}+m_{h} g\right\}
$$

where each $H_{i}$ is a coset of $H=[g]$,

$$
S^{\prime \prime}=\left\{a_{1}, a_{1}+g, \ldots, a_{1}+m_{1} g, \ldots, a_{h}, a_{h}+g, \ldots, a_{h}+m_{h} g\right\}
$$

does not contain a whole coset of $H, a_{i}+\left(m_{i}+1\right) g \neq a_{j}$ (mod $n$ ) for any $i, j=1, \ldots, h, 1 \leq a_{1}<a_{2}<\ldots<a_{h}<n$, and

$$
|(S+g) \cap S|=k-h \geq\left|\left(S+g^{\prime}\right) n S\right| \text { for every } g^{\prime} \pm 0 \text { in } G \text {. }
$$

Let $X=\left\{a_{1}, a_{2}, \ldots, a_{h}\right\}$. Then

$$
y=\left\{a_{1}+\left(m_{1}+1\right) g, \ldots, a_{h}+\left(m_{h}+1\right) g\right\}=g-X,
$$

since $-S^{\prime \prime}=S^{\prime \prime}$. By a similar method we can show that (3) holds good. Suppose that $h \geq 3$. If for each $j=1, \ldots, h, X+a_{j}=Y=g-X$, then $h=|X|=|[X-X]|=p$, and this divides $n$, and (6) also holds good. We have then $a \equiv(2 s+1) g(\bmod q)$. Now if $\left|\cup H_{i}\right| \neq 0$, then $H \cap S=\emptyset$. We note that the number of elements of $a_{i}$ in $X$ that belong to a particular coset $H_{i}$ of $H$ and the number of $a_{j}$ in $X$ that belong to $-H_{i}$ are the same, therefore since $p$ is odd, there is at least one $a_{i} \in X$ such that $a_{i} \in H$ which contradicts the fact that $H \cap S=\varphi$. Hence in this case, $\left|\cup H_{i}\right|=0$ and $H \cap S \neq \varphi$. Now if $(g, q)=d>1$, then $d \mid a$ and thus $d$ divides each element in $S$ which is impossible. Hence $(g, p)=g$ and $q \leq n / g$. It is then clear that each $m_{i}<q-1$. Otherwise for some $i$ with $I \leq i \leq p$, 
$a+(g+i-1) q \in S$ will be one of the elements of $S$ that belong to $\left\{a+(i-1) q+g, \ldots, a+(i-1) q+m_{i} g\right\}$ or $a+(g+i-1) q=a+(i-1) q+\left(m_{i}+1\right) g$, which is not true. From this, it can be shown that each of the cosets $K_{i}$ of $K=[q]$ which is contained in $S$ is of the form $a+i g+K$, $i<q-1$. Since $3 a \equiv g$ (mod $q)$, we have $3 a-g=2 q$ if $g<q$ and $g-3 a=x q$ if $g>q$ where $x \equiv 1 \quad(\bmod 3)$. Now since $a+K \subseteq S$, therefore $-a+K \subseteq S$. We have $-a+K=a+(g+2 q) / 3-g+K$ if $g<q$ and $-a+K=a+(g-x q) / 3-g+K$ if $g>q$. But neither $(g+2 q) / 3$ nor $(g-x q) / 3$ is of the form $i g, 1 \leq i \leq q-1$, for otherwise $g$ will divide $q$.

By a similar method and the proof of Lemma 4, we can show that all the other possibilities cannot occur. Hence $h \leq 2$. If $h=1,2$, then using the proof of Lemma 4 again, we can show that these cases cannot occur also. Hence the possibility that $|(S+g) n S|$ is maximal for some $g$ with $(g, n)>1$ is excluded.

This is the end of the proof of Theorem 2 .

\section{References}

[1] Palahenedi Hewage Diananda and Hian Poh Yap, "Maximal sum-free sets of elements of finite groups", Proc. Japan Acad. 45 (1969), 1-5.

[2] P. Erdös, "Extremal problems in number theory", Proc. Sympos. Pure Math. 8, 181-189 (Amer. Math. Soc., Providence, Rhode Island, 1965).

[3] J.H.B. Kemperman, "On small sumsets in an abelian group", Acta Math. 103 (1960), 63-88.

[4] Henry B. Mann, Addition theorems: The addition theorems of group theory and number theory (Interscience Tracts in Pure and Applied Mathematics, Number 18, John Wiley, New York, London, Sydney, 1965).

[5] A.H. Rhemtulla and Anne Penfold Street, "Maximal sum-free sets in finite abelian groups", Bull. Austral. Math. Soc. 2 (1970), 289-297. 
[6] A.H. Rhemtulla and Anne Penfold Street, "Maximal sum-free sets in elementary abelian p-groups", Canad. Math. Bulz. (to appear).

[7] H.P. Yap, "The number of maximal sum-free sets in $C_{p}$ ", Nanta Math. $2(1968), 68-71$.

[8] H.P. Yap, "Structure of maximal sum-free sets in $C_{p}$ ", Acta Arith. $17(1970), 29-35$.

[9] Hian Poh Yap, "Structure of maximal sum-free sets in groups of order $3 p "$ " Proc. Jcipan Acad. 46 (1970), 758-762.

[10] H.P. Yap, "Maximal sum-free sets in finite abelian groups", Buzz. Austral. Math. Soc. 4 (1971), 217-223.

University of Alberta,

Edmonton,

Canada. 